\title{
Wavelets-Computational Aspects of Sterian Realistic Approach to Uncertainty Principle in High Energy Physics: A Transient Approach
}

\author{
Cristian Toma \\ Faculty of Applied Sciences, Politehnica University, 313 Splaiul Independentei, 060042 Bucharest, Romania \\ Correspondence should be addressed to Cristian Toma; cgtoma@physics.pub.ro
}

Received 13 August 2013; Accepted 21 August 2013

Academic Editor: Carlo Cattani

Copyright (C) 2013 Cristian Toma. This is an open access article distributed under the Creative Commons Attribution License, which permits unrestricted use, distribution, and reproduction in any medium, provided the original work is properly cited.

\begin{abstract}
This study presents wavelets-computational aspects of Sterian-realistic approach to uncertainty principle in high energy physics. According to this approach, one cannot make a device for the simultaneous measuring of the canonical conjugate variables in reciprocal Fourier spaces. However, such aspects regarding the use of conjugate Fourier spaces can be also noticed in quantum field theory, where the position representation of a quantum wave is replaced by momentum representation before computing the interaction in a certain point of space, at a certain moment of time. For this reason, certain properties regarding the switch from one representation to another in these conjugate Fourier spaces should be established. It is shown that the best results can be obtained using wavelets aspects and support macroscopic functions for computing (i) wave-train nonlinear relativistic transformation, (ii) reflection/refraction with a constant shift, (iii) diffraction considered as interaction with a null phase shift without annihilation of associated wave, (iv) deflection by external electromagnetic fields without phase loss, and (v) annihilation of associated wave-train through fast and spatially extended phenomena according to uncertainty principle.
\end{abstract}

\section{Introduction}

According to the Sterian realistic approach [1], one cannot make a device for the simultaneous measuring of the canonical conjugate variables in the conjugate Fourier spaces.

Generally, Heisenberg's uncertainty principle states that incompatible dynamic variables in relation to the measuring process satisfy the following relations:

$$
\begin{aligned}
& \Delta x \Delta p_{x} \geq \frac{\hbar}{2}, \\
& \Delta y \Delta p_{y} \geq \frac{\hbar}{2}, \\
& \Delta z \Delta p_{z} \geq \frac{\hbar}{2}, \\
& \Delta t \Delta E \geq \frac{\hbar}{2},
\end{aligned}
$$

where $\Delta x, \Delta y$, and $\Delta z$ are the uncertainties in position for each spatial axis, $\Delta p_{x}, \Delta p_{y}$, and $\Delta p_{z}$ are the uncertainties in momentum, and $\hbar$ is the reduced Planck constant, thus leading to the following statement. The product of the inaccuracies arising from simultaneous determination of two canonical conjugate variables is of the order of magnitude of Planck's constant (see [2] for more details).

These relations can be written also in the form of commutation relationships between corresponding incompatible observables according to the theorem: being given two hermitic operators $\widehat{A}$ and $\widehat{B}$ and their commutator $\widehat{C}$, one can demonstrate the relationship

$$
\Delta A \Delta B \geq \frac{\langle\widehat{C}\rangle}{2},
$$

where the brackets for $\widehat{C}$ indicate an expectation value (aspect emphasized in [1]). By this moment, no actual physical measurements can avoid the limitations of these relationships.

The Sterian realistic approach is based on the analysis of a wave packet (the usual mathematical model for propagating associated wave function). From the classical theory of wave propagation, it is known that the width of a wave packet $\Delta x$ involves a certain width $\Delta k$ in the reciprocal Fourier 
transform space, which corresponds to the wave vector $k$. Standard Fourier analysis involves

$$
\Delta x \Delta k \geq 2 \pi \text {. }
$$

Thus uncertainties for $\Delta x$ and $\Delta k$ are interconnected. Since momentum $p$ for a quantum particle is proportional to the wave vector of the associated wave $(p=\hbar k)$, it results in that a lower value $\Delta x$ involves a greater value $\Delta p$ for uncertainty upon momentum. Similarly, for finite duration disturbances, the standard Fourier analysis involves

$$
\Delta t \Delta \omega \geq 2 \pi
$$

against reciprocal Fourier transform spaces of a pair of signals, where $\Delta \omega$ corresponds to the spectral width.

Further it is considered that in quantum physics simultaneous measurements of the canonical conjugate variables (as space momentum, time energy) cannot be performed, because these variables correspond to the reciprocal Fourier spaces. The time interval required by any device for performing any measurement upon a certain quantum particle or system cannot be avoided. The standard experiments are not meant for simultaneous measurements of position and momentum of a quantum system, as it is usually admitted, being a consequence of the used measurement device (Fourier transformer), through which the signals pass having a finite speed. Any quantum system is subject to uncertainty relations, proving its dual nature. Due to the requirements of the principle of causality in the theory of relativity, one cannot make a device for simultaneous measuring of the canonical conjugate variables in the conjugate Fourier spaces. Due to finite speed of propagation of interactions, signal switching within physical system to perform Fourier transform has a finite duration. Moreover, uncertainty relations are considered to confirm the principle of causality due to the finite speed of propagation of interactions required by any measurement process.

However, this approach is far from being rigorous. Specific aspects regarding relativistic transformation of waves implied by measuring procedures should be added. Next a rigorous analysis for coherence aspects implied by different interaction phenomena should be performed (the Fourier transformation being just a mathematical tool for analyzing this coherence for wave packets corresponding to a certain quantum particle). Finally, aspects regarding creation annihilation of quantum particles during the measurement process should be analyzed by taking into consideration correlation aspects in quantum field theory and the change from coordinate space to momentum space (reciprocal Fourier spaces) required by the mathematical model.

\section{Supplementary Aspects regarding Relativistic Wavelets Transformation}

As has been shown in [3], a certain wave function received by a reference system $S$ (represented by a material medium) is transformed according to Lorentz transformation as

$$
\phi^{\prime}\left(x^{\prime}, y^{\prime}, z^{\prime}, t^{\prime}\right)=L \phi(x, y, z, t),
$$

where the following hold. (i) The space-time coordinates $x, y, z$, and $t$ corresponding to the received wave are transformed into the $x^{\prime}$, $y^{\prime}, z^{\prime}$, and $t^{\prime}$ coordinates of the transformed wave according to the action of the Lorentz transformation matrix $L$ upon the cuadrivector $[x, y, z, i t]^{T}$ of this supposed coordinates $x, y, z$, and $t$ (the coordinates the wave would have had in the absence of interaction), with the space-time origin considered in the point of space and at the moment of time where the received wave first time interacts with the observer's material medium (in fact $L$ matrix multiplies the column cuadrivector $[x, y, z, i t]^{T}$ so as to result in the column cuadrivector $\left.\left[x^{\prime}, y^{\prime}, z^{\prime}, t^{\prime}\right]^{T}\right)$.

(ii) The transformed wave function $\phi^{\prime}$ is represented by a vector or a higher-order tensor which describes the quantum field/particle. For an electromagnetic wave, $\phi$ corresponds to the cuadridimensional vector $[A, i V]^{T}$. In the most general case, $\phi$ corresponds to a state vector describing the quantum particle. The Lorentz matrix $L$ multiplies the vector of higher-order tensor $\phi$ of the received wave so as to result in the vector or higher-order tensor $\phi^{\prime}$ of the transformed wave.

As a consequence, each Lorentz transformation is specific to a certain wave train, with the zero moment of time considered when the received wave first time interacts with the observer's material medium. Thus this relativistic transformation is connected to transient phenomena (as the propagation of associated waves), and no memory of previous measurements is involved regarding space-time measurements for events in different reference (material) systems. Logical contradictions as clock paradox do not appear any more.

This aspect implies a very important property of measuring device to be added to Sterian realistic approach, with significant consequences upon computational methods: the observer's material medium acts in a nonlinear manner upon a superposition of received wave trains $\phi_{i}$ in a certain area. Each wave train has its own amplitudes, frequency, and wave vector, but it has also its own zero moment of time to be considered within Lorentz transformation. Thus at a certain moment of time there will be different time intervals $\Delta t_{i}$ for each wave train considered from each specific time origin $T_{0 i}$ as

$$
\Delta t_{i}=T-T_{0 i}
$$

According to Lorentz transformation of space-time coordinates, the time interval $\Delta t_{i}^{\prime}$ for the wave train transformed by the observer's material medium (considered from this specific time origin $T_{0 i}$ ) will be

$$
\Delta t_{i}^{\prime}=\frac{1}{\sqrt{1-(v / c)^{2}}} \Delta t_{i}
$$

Thus all parts of the wave trains $\phi_{i}$ received by the observer's material medium at a certain moment of time $T$ will be translated in time with different values, depending on these 
time differences $\Delta t_{i}^{\prime}$ considered from different time origins $T_{0 i}$ as

$$
\phi_{i}(T) \longrightarrow \phi_{i}\left(T_{0 i}+\Delta t_{i}^{\prime}\right)
$$

(a certain delay time; there is no possible anticipation of a future event). This translation is a nonlinear transformation, since parts of the reviewed wave train are translated differently. After this translation is performed, it can be considered that the Lorentz matrix $[L]$ acts in a linear manner upon the vector or higher-order tensor specific to the wave train $\phi_{i}$ so as to result in the final transformed wave train $\phi_{i}^{\prime}$ as

$$
\phi_{i}^{\prime}\left(T_{0 i}+\Delta t_{i}^{\prime}\right)=[L] \phi_{i}\left(T_{0 i}+\Delta t_{i}^{\prime}\right) .
$$

Thus it results in that high energy (relativistic) corrections regarding finite speed for propagation of interaction for measurements based on quantum aspects (presented in Sterian realistic approach) should be completed with considerations about nonlinear transformations for superposition of wave trains (as shown above) so as to result in a rigorous and complete transient approach. With each wave train being described by specific frequency and wave vector, it results in that temporal and spatial correlations are involved in these relativistic transformations for part of wave trains. Supplementary aspects regarding phase will be presented in the next paragraph.

\section{Aspects regarding Phase Changes for Sterian Realistic Approach}

The most usual transformations performed by a certain material medium upon a wave function are reflection and refraction. A preliminary analysis of reflection/refraction phenomena is based on classical electromagnetic field, which corresponds in fact to the wave function associated with a photon (the electric field $\mathbf{E}$, magnetic field $\mathbf{B}$, vector potential $\mathbf{A}$, and scalar potential $\mathbf{V}$ are the main quantities used). This wave function can explain basic aspects in wave theory of light as reflection/refraction angles and the influence of polarization upon electric and magnetic fields for both reflected and refracted beams.

At first view this classical model is just an approximation, since according to the rigorous quantum theory the energy can be transmitted just in a discontinuous manner, with amounts of $\hbar \omega$. Parts of the incident (received) wave undergo an interaction with a certain interface, so quantum aspects should be involved. However, as was shown in [4], an analysis based exclusively on standard quantum aspects cannot explain important phase aspects. Within such a model, light consists of photons which are packets of energy that primarily interact with interface atoms. Through this interaction, the energy of the photon is absorbed by collectivised electrons of the solid crystalline lattice, and the photon ceases to exist. Then the electron will return to a lower energy state by emitting a photon. Each photon behaves more like a point source, as if the light was originating right there. These emitted spherical waves generate the total wavefront as the envelope that encloses all these point-source waves.
The effect of interface nonuniformities could be considered as vanishing by drawing a tangent line as a global approximation through the front surface for each point-source wave. Yet there is no valid argument regarding a minimum value for the radii of curvature of this tangent line. Theoretically, it could be very small, and thus the global tangent line could consist of a lot of local curves with significant curvatures which are joined together. In this way a lot of divergent light beams could be created along the reflected/refracted trajectory, and the directionality would be lost very quickly.

A more rigorous standard quantum model considers that photons interact with collectivised electrons of the solid crystalline lattice before being reemitted. Since the associated wave function for the collectivised electrons is represented in position for large space intervals, the influence of local nonuniformities is decreased. Thus a tangent line local radius of curvature greater than a certain value can be drawn, and a better directionality for reflected/refracted wave can be obtained.

However, this standard quantum model does not take into account the phase shift between the incident and the reemitted wave for different points of the interface. A complete analysis based on quantum theory should consider that waves reemitted from different points of the interface are part of the wavet rain corresponding to a certain photon, with the probability of detecting a reflected/refracted photon being determined by the coherent plane-wave compounding method (it is well known that a particle interferes just with itself). There is no valid argument based exclusively on standard quantum theory regarding the constant phase shift between the local incident wave and the corresponding local reemitted wave in each interface point. The wave function for collectivised electrons of the crystal lattice is far from being constant in space time along this interface.

For this reason, space correlations for the incident wave train and the reemitted wave train should be taken into account. This could suggest that lattice quantum functions interact in a global manner with parts of the incident wave on a large spatial area of the interface, with a certain correlation between phases of reflected/refracted wave being noticed even for surface points separated by crystal defects. Some support functions generated on this interface able to correlate the phase of reemitted waves by remote interface points could be taken into consideration for a complete computational model.

In the same study [4] showed that space correlations achieved within a very short time interval for nonadjacent spatial intervals which interact with wavefronts (part of the reflected/refracted wave) become a key issue within an exclusively quantum model if we consider that reflected/refracted wave can undergo diffraction at a later time. The requirement of constant phase shift for parts of the associated wave generated by points or edges of a diffraction grating is still valid-yet the points or edges of such a diffraction grating are situated on nonadjacent areas, so they cannot be correlated by any surface quantum wave functions.

These aspects imply either the use of space-propagation properties for the support functions previously mentioned or the use of the assumption that the reemitted wavefronts 
are in phase with the received wavefronts (parts of the same incident wave), with the phase shift being null anywhere, at any time.

The second choice is far more attractive (being more simple and connected to quantum field theory). However, it implies the extension of phase conservation from reception/ emission of associated waves to reception/emission of parts of the same associated wave. Moreover, it requires a distinction between these reflection/refraction or diffraction phenomena (which does not alter coherence, but modifies momentum) and the annihilation/creation phenomena involved by any interaction in quantum field theory; otherwise any reflection/refraction or diffraction of a part of the incident wave (considered as a sequence annihilation-creation) would generate the annihilation of the entire associated wave function in any other point of space (the wave function vanishes instantaneously).

However, the use of some support functions for computational aspects (similar to wavelets presented in $[5,6]$ ) is still required in certain circumstances, usually when the properties of the material medium imply a nonzero phase shift (the same in any point where the wave function interacts with it). An example is represented by the $\pi$ phase shift (corresponding to $1 / 2$ of the wavelength) for reflection on metallic surfaces, when the electric field $\mathbf{E}$ should vanish very quickly, in any point of the metallic surface, such that all parts of the wave function are to be shifted by $\pi$ before spatial reflection.

For this reason a complete computational model cannot be based exclusively on standard quantum aspects. Certain support functions corresponding to spatial coherence on extended space intervals should be added for a correct computation of wave trajectories in case of reflection/refraction or diffraction phenomena. Moreover, the assumption regarding nonannihilation of the entire wave function when certain parts of it undergo interaction with phase conservation (or even constant phase shift) should be also added for a complete model. These phase aspects should be added to Sterian realistic approach for correct determination of wave space properties (as the wave vector or the momentum of the associated wave) in a more general and rigorous transient approach. At this moment, Sterian realistic approach uses just spatial selection for computing the wave vector (a certain direction is selected) without taking into consideration phase properties.

\section{Implications of Using Quantum Field Theory upon the Realistic Approach}

Any measurement for the wave vector (proportional to the momentum of the quantum particle) cannot be based exclusively on the spatial selection of certain directions (as is considered in the Sterian realistic approach in an implicit manner, due to the use of Fourier space). Any direction of propagation generated by a diffraction grating, for example, implies a validation by means of a final interaction with the material medium (the particle is annihilated while it interacts, generating a specific signal for the measurement device).

First we must take into account the possibility for the particle to be deflected or captured (for a very short time interval) by the electric or magnetic field of the measurement device. As was shown in [7], the standard second quantification cannot describe these interactions without the use of certain support functions. The use of virtual photons for analyzing the trajectory of an electron generates the phenomenon of phase loss due to multiple interactions to be computed along the trajectory. For example, an electrostatic field should be decomposed (using the Fourier transformation) in a set of waves with a certain angular frequency $\omega$ and a certain wave vector $k$ :

$$
A_{\mu}^{e}\left(x_{0}, \vec{x}\right)=\frac{1}{(2 \pi)^{2}} \int d q A_{\mu}^{e}(q) e^{-i q x},
$$

where the product $q x$ stands for

$$
q x=q_{0} x_{0}-\vec{q} \vec{x}
$$

with $x_{0}$ representing the time coordinate, $\vec{x}=\left(x_{1}, x_{2}, x_{3}\right)$ representing the vector of position, $q_{0}$ representing the angular frequency, and $\vec{q}=\left(q_{1}, q_{2}, q_{3}\right)$ representing the wave vector. The measurement system is chosen so as $c=1, h /(2 \pi)=$ 1 for performing a better correspondence from the angular frequency to the energy and from the wave vector to the momentum of the quantum particle (see [8] for more details).

The quantities $A_{\mu}^{e}(x)$ and $A_{\mu}^{e}(p)$ do not correspond to standard photons, and for this reason they cannot be substituted by operators as required by second quantification theory. However, experimental facts have shown that the electromagnetic field effect can be studied using the perturbations method from quantum theory.

Using this method [8] the matrix element (for the first order of perturbation) corresponding to the electromagnetic interaction between the electron and the electromagnetic field is represented by

$$
\begin{aligned}
& S_{f i}^{(1)} \\
& =-i e \int d x \bar{v}_{r^{\prime}}^{(+)}\left(\vec{p}_{f}\right) e^{i p_{f} x} \int d q A_{\mu}^{e}(q) \gamma_{\mu} e^{-i q x} v_{r}^{(-)}\left(\vec{p}_{i}\right) e^{-i p_{i} x},
\end{aligned}
$$

where $v_{r}^{( \pm)}$stands for the basic vectors of an electron with positive or negative frequencies (energies) - usually column vectors, $\bar{v}_{r}^{( \pm)}$stands for their Dirac conjugate vectors-usually line vectors, index $r$ denotes the projection of the spin along a certain axis, and $\vec{p}_{f}$ and $\vec{p}_{i}$ correspond to the final and initial three-dimensional momenta for the electron, respectively.

These can be also written as

$$
S_{f i}^{(1)}=-i e \int d q \bar{v}_{r^{\prime}}^{(+)}\left(\vec{p}_{f}\right) \gamma_{\mu} A_{\mu}^{e}(q) v_{r}^{(-)}\left(\vec{p}_{i}\right) \delta\left(q-p_{f}+p_{i}\right) \text {. }
$$

In both previous equations the expression $A_{\mu}^{e} \gamma_{\mu}$ corresponds to the sum

$$
A_{\mu}^{e} \gamma_{\mu}=A_{0}^{e} \gamma_{0}+\sum_{n=1}^{3} A_{n}^{e} \gamma_{n}
$$


where $A_{\mu}^{e}$ represents the cuadrivector of the electromagnetic field and $\gamma_{\mu}$ represents the Dirac matrices (its argument corresponds to the energy and momentum conservation laws).

If the electromagnetic field does not depend on time, the cuadripotential $A_{\mu}^{e}(x)$ can be presented as

$$
A_{\mu}^{e}(x)=A_{\mu}^{e}(\vec{x})=\frac{1}{(2 \pi)^{3 / 2}} \int d q e^{i \vec{q} \vec{x}} A_{\mu}^{e}(\vec{q})
$$

which shows that the virtual photons composing the electromagnetic field have a nonzero value just for the momentum (the energy corresponding to quantity $q_{0}$ is equal to zero). As a consequence, the matrix element for the first order of perturbation can be written as

$$
\begin{aligned}
S_{f i}^{(1)}= & -i e \int d q \bar{v}_{r^{\prime}}^{(+)}\left(\vec{p}_{f}\right) \gamma_{\mu} A_{\mu}^{e}(\vec{q}) v_{r}^{(-)}\left(\vec{p}_{i}\right) \\
& \times \delta\left(\vec{q}-\vec{p}_{f}+\vec{p}_{i}\right) \delta\left(p_{f 0}-p_{i 0}\right) .
\end{aligned}
$$

By performing the integration on $d q$, it results in

$$
S_{f i}^{(1)}=-i e \bar{v}_{r^{\prime}}^{(+)}\left(\vec{p}_{f}\right) \gamma_{\mu} A_{\mu}^{e}(\vec{q}) v_{r}^{(-)}\left(\vec{p}_{i}\right) \delta\left(p_{f 0}-p_{i 0}\right) .
$$

According to standard interpretation of quantum field theory, this first order element from perturbation method is connected to the probability of an interaction between an electron with initial momentum $\vec{p}_{i}$ and energy $p_{i 0}$ and a virtual photon with momentum $\vec{q}$ so as to result in an electron with momentum $\vec{p}_{f}$ and energy $p_{f 0}$.

However, for computing the whole interaction for an electron in an electrostatic field (so as to determine the trajectory of the associated wave train) we have to consider that the electron has to undergo multiple interactions with such virtual photons until the action of the exterior field vanishes (such multiple interactions are allowed by second quantification theory). According to the quantum laws, each interaction transforms the initial electron (its wave train used in computation) into a new wave train (the final electron) with different characteristics (another momentum and energy) and so on.

Considering that the wave train corresponding to the electron undergoes a set of interactions with virtual photons, it results in that a certain transient time is required by each interaction, so as physical quantities as wavelength and angular frequency to be transferred in this local phenomenon (these quantities are used for further computing the matrix elements for creation/annihilation phenomena). This transient time causes a phase loss for the initial wave train, so it results in that the great number of interactions of the initial electron in an electrostatic (Coulombian) field would finally cause significant phase loss (the time length of the wave train associated with the final electron tends to zero). This corresponds to a vanishing phenomenon for the initial electron, in contradiction with experimental facts.

This phenomenon can be avoided into a complete and rigorous transient approach if the whole trajectory is computed using just one Lagrangian function for the whole interaction of the electron with the electromagnetic field, according to the dynamical equations:

$$
\begin{gathered}
\left(-\frac{\partial^{2}}{\partial t^{2}}+\nabla^{2}\right) A_{\mu}(x)=-e \bar{\psi} \gamma_{\mu} \psi \\
\left(i \gamma_{\mu} \frac{\partial}{\partial x_{\mu}}-e \gamma_{\mu} A_{\mu}\right) \psi(x)-m \psi(x)=0, \\
\bar{\psi}(x)\left(i \gamma_{\mu} \frac{\partial}{\partial x_{\mu}}+e \gamma_{\mu} A_{\mu}\right)+m \bar{\psi}(x)=0
\end{gathered}
$$

(see [8]). Thus the wave function corresponding to the electron is modified in a continuous manner along its trajectory (according to the first quantification theory). The exterior field acts as a support function (as mentioned in previous paragraph), determining the evolution of the electron wave train. Moreover it shows the need for a certain reference system which acts upon the wave trains corresponding to quantum particles and their associated waves in any rigorous computational model for transient phenomena (the exterior electromagnetic field is part of it). This aspect is supported by the higher magnitude and a slow time variation of these exterior electromagnetic fields and by the lack of reversibility for diffraction phenomena in quantum physics (we can notice an electron diffraction phenomenon when an electron beam interacts with a motionless crystal lattice, but we cannot imagine a diffraction of an entire crystal lattice when it interacts with a motionless spatial distribution of electrons).

Similar to reflection/refraction and diffraction phenomena previously presented, these deflections do not alter coherence. They just alter the spatial directions (the wave vector) of certain wave trains previously selected (e.g., by a diffraction grating). After corrections due to possible deflections are computed, we should analyze the annihilation phenomenon which validates the detection of a particle (wave train) with certain characteristics. The rigorous model is based on quantum field theory (no virtual particles are taken into consideration). According to this model, the wave train is analyzed using the momentum space. The switch from position space to momentum space is performed using the Fourier transformation. The space and time origins are selected in this point of interaction, when the associated wave train is received by the material medium. The annihilation of the particle implies the instant annihilation of all parts of this wave train, irrespective of the distance to this interaction point. It is true that this seems to contradict the relativity postulated (no speed can surpass the light speed), but in fact any speed can be noticed just by an emission and a reception of a certain signal or particle. The annihilation of a certain quantum particle cannot be noticed in two different space areas. Thus there is no contradiction. Moreover, the existence of some high speed support functions which cancel the wave in an instant manner can be supported by uncertainty principle also. Since the particle ceases to exist, its final momentum is zero. Being no uncertainty, it results in that

$$
\Delta p_{x}=\Delta p_{y}=\Delta p_{z}=0,
$$


and, according to uncertainty principle,

$$
\begin{aligned}
& \Delta x \geq \frac{\hbar}{\Delta p_{x}} \longrightarrow \infty, \\
& \Delta y \geq \frac{\hbar}{\Delta p_{y}} \longrightarrow \infty \\
& \Delta z \geq \frac{\hbar}{\Delta p_{z}} \longrightarrow \infty
\end{aligned}
$$

Thus this annihilation phenomenon can be considered as being infinitely extended. Unlike standard phenomena considered by Sterian realistic approach, the uncertainty for momentum determines the uncertainty for space intervals. However, for computational aspects it can be simply noticed that discontinuities cannot be analyzed using the differential form of wave equations. A discontinuity looks like a limitation, but physically they are represented by fracture phenomena for certain quantities. An avalanche process can be easily imagined and computed (similar to mechanical engineering).

\section{Conclusions}

This study has presented supplementary aspects for the Sterian realistic approach to uncertainty principle. It was shown that high energy (relativistic) corrections regarding finite speed for propagation of interaction for measurements based on quantum aspects should be completed with considerations about nonlinear transformations for superposition of wave trains so as to result in a rigorous and complete transient approach. With each wave train being described by a specific frequency, wave vector, and its own Lorentz transformation (with specific time origin), it results in that temporal and spatial correlations are involved in these relativistic transformations for part of wave trains.

It was also shown that a complete computational model for a transient approach cannot be based exclusively on standard quantum aspects. Certain support functions corresponding to spatial coherence on extended space intervals (similar to wavelets propagation in composite materials [9] or to multiscale phenomena [10]) should be added for a correct computation of wave trajectories in case of reflection/refraction or diffraction phenomena. Moreover, an assumption regarding nonannihilation of the entire wave function when certain parts of it undergo interaction with phase conservation (or even constant phase shift) should be also added for a complete model. The need for a certain exterior reference system acting upon the quantum wave train is also presented (based on some diffraction aspects).

Computational aspects for deflection of associated waves in electromagnetic fields were also studied. It emphasized the need for a certain reference system which acts upon the wave trains corresponding to quantum particles and their associated waves in any rigorous computational model for transient phenomena (the exterior electromagnetic field is part of it as support functions), so that phase-loss phenomenon generated by the use of second quantification is to be avoided.

Finally it was shown that both the switch from position space to momentum space and the annihilation of a certain quantum particle are represented by instant phenomena supported by uncertainty principle (unlike considerations in Sterian realistic approach); since there is no uncertainty for final momentum when the particle is annihilated, an infinitely extended phenomenon is generated (an instant propagating spatial noise is generated, similar to temporal Gaussian noise-see [11], and frequency dependent noisesee [12]).

\section{References}

[1] P. E. Sterian, "Realistic approach of the relations of uncertainty of Heisenberg," Advances in High Energy Physics, vol. 2013, Article ID 872507, 7 pages, 2013.

[2] L. D. Landau and E. M. Lifshitz, Quantum Mechanics: Non-Relativistic Theory, vol. 3, Pergamon Press, Oxford. UK, 3rd edition, 1974.

[3] E. G. Bakhoum and C. Toma, "Relativistic short range phenomena and space-time aspects of pulse measurements," Mathematical Problems in Engineering, vol. 2008, Article ID 410156, 20 pages, 2008.

[4] E. Bakhoum and C. Toma, "Transient aspects of wave propagation connected with spatial coherence," Mathematical Problems in Engineering, vol. 2013, Article ID 691257, 5 pages, 2013.

[5] C. Cattani, "Fractional calculus and Shannon wavelet," Mathematical Problems in Engineering, vol. 2012, Article ID 502812, 26 pages, 2012.

[6] J. Leng, T. Huang, and C. Cattani, "Construction of bivariate nonseparable compactly supported orthogonal wavelets," Mathematical Problems in Engineering, vol. 2013, Article ID 624957, 11 pages, 2013.

[7] E. G. Bakhoum and C. Toma, "Mathematical transform of traveling-wave equations and phase aspects of quantum interaction," Mathematical Problems in Engineering, vol. 2010, Article ID 695208, 15 pages, 2010.

[8] N. Nelipa, Physique des Particules Elementaires, Editions MIR, Moscou, Russia, 1981.

[9] J. J. Rushchitsky, C. Cattani, and E. V. Terletskaya, "Wavelet analysis of the evolution of a solitary wave in a composite material," International Applied Mechanics, vol. 40, no. 3, pp. 311-318, 2004.

[10] C. Cattani, "Multiscale analysis of wave propagation in composite materials," Mathematical Modelling and Analysis, vol. 8, no. 4, pp. 267-282, 2003.

[11] M. Li and W. Zhao, "On bandlimitedness and lag-limitedness of fractional Gaussian noise," Physica A, vol. 392, no. 9, pp. 19551961, 2013.

[12] M. Li and W. Zhao, "On 1/f noise," Mathematical Problems in Engineering, vol. 2012, Article ID 673648, 23 pages, 2012. 

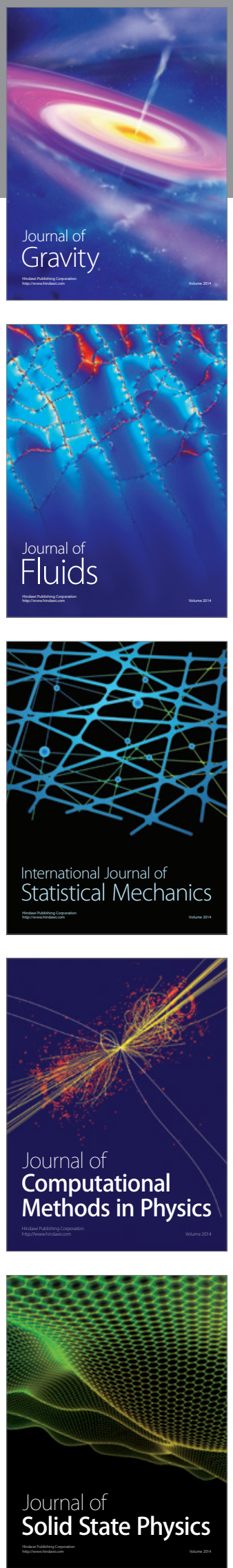

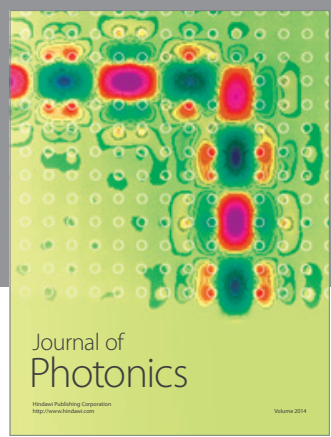

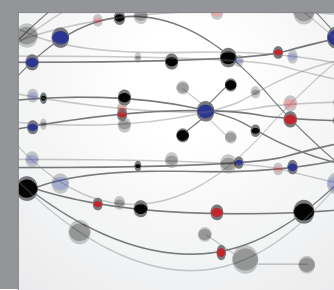

The Scientific World Journal

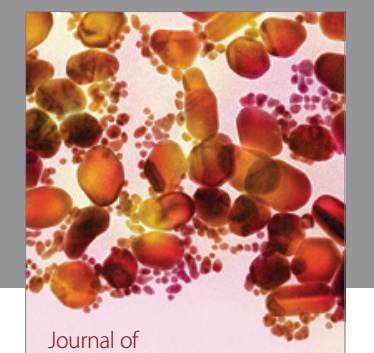

Soft Matter
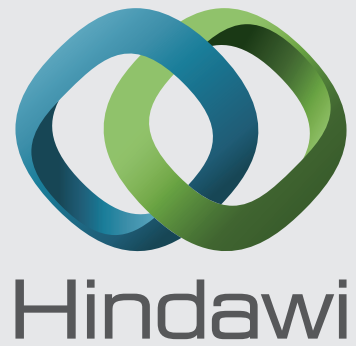

Submit your manuscripts at

http://www.hindawi.com
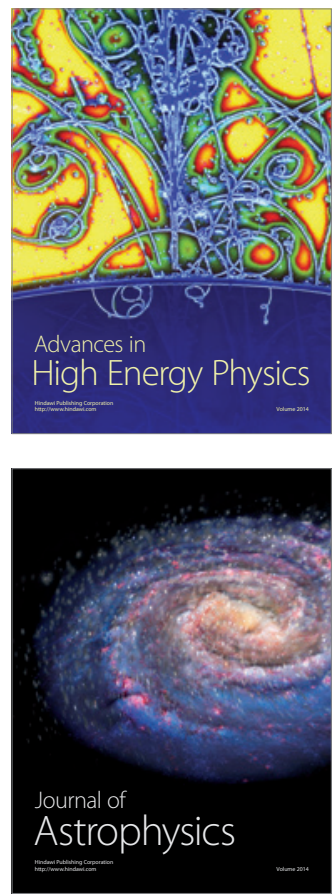
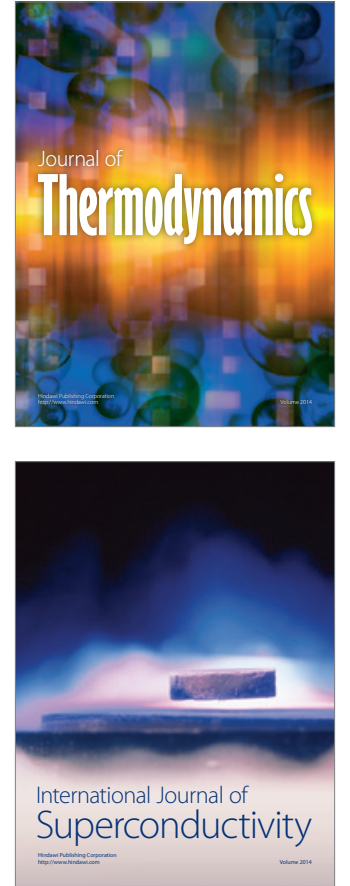
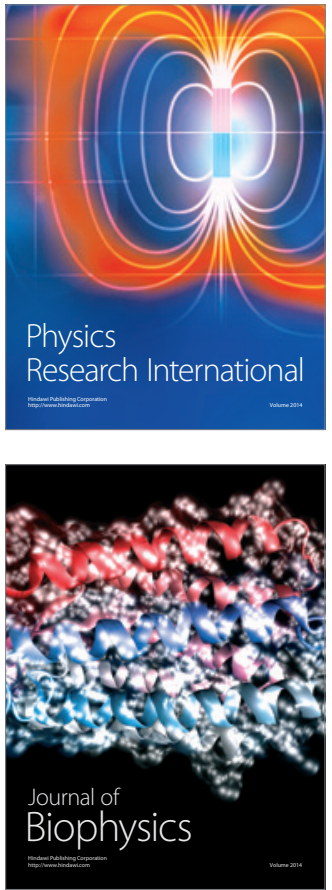
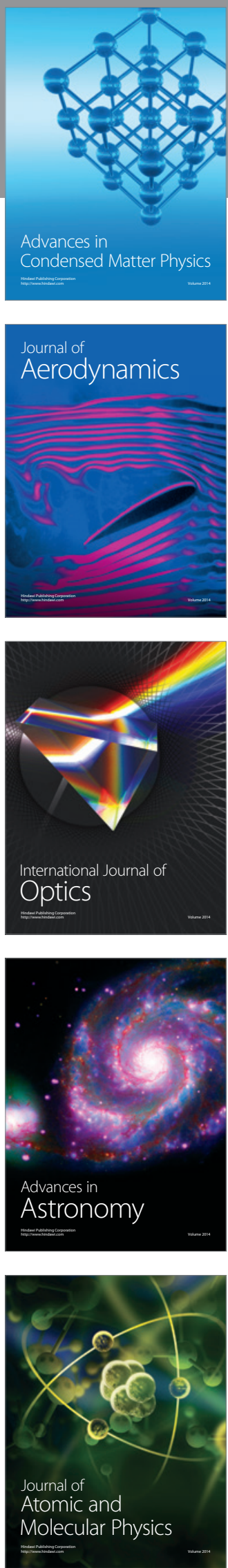